\title{
AVALIAÇÃO POSTURAL E DO SONO DE BOMBEIROS MILITARES DA CIDADE DE CAJAZEIRAS-PB
}

\author{
Wagner Beserra da Cunha ${ }^{1}$ \\ Hiago Pinheiro ${ }^{2}$ \\ Juliane Carla Medeiros de Sousa ${ }^{3}$ \\ Luciano Braga de Oliveira ${ }^{4}$ \\ Elisangela Vilar de Assis ${ }^{5}$ \\ Michel Jorge Dias ${ }^{6}$
}

RESUMO: OBJETIVO: Avaliar a postura e os aspectos de sono dos bombeiros militares da cidade de Cajazeiras-PB. Método: Trata-se de um estudo analítica, transversal com abordagem quantitativa. Foi composto por 20 bombeiros militares do $5^{\circ}$ batalhão do município de Cajazeiras - PB, a amostra se deu por conveniência e de forma aleatória, sendo desenvolvida nos meses de agosto e setembro de 2018. Foi utilizado um questionário elaborado pelos pesquisadores contendo dados sobre o perfil sócio demográfico. Para avaliação do sono utilizou-se o questionário de qualidade do sono de Pittsburgh (PSQI) e para a análise postural foi utilizado os dados do programa AVAESPORTE, que é um software de avaliação física e prescrição de treino. Os testes de Kolmogorov-Smirnov, Shapiro Wilk e Levene foram utilizados para verificar a normalidade e homogeneidade dos dados. Resultados: Observou a predominância em vários segmentos das alterações posturais, onde as mais predominantes foram: inclinação à direita da cabeça $25 \%$, cabeça projetada para frente $50 \%$, elevação do ombro à esquerda $65 \%$, protrusão do ombro $50 \%$, escápulas aladas $60 \%$, cristas ilíacas assimétricas $80 \%$, anteversão pélvica $65 \%$ e hipercifose torácica com $55 \%$. O índice geral encontrado indica que os bombeiros militares possuem uma qualidade de sono alterada, ficando dentro da categoria ruim, onde prevaleceu a categoria de distúrbios de sono com uma porcentagem de $40 \%$. Conclusão: O estudo evidenciou alterações posturais em

\footnotetext{
${ }^{1}$ Graduando do curso Bacharelado em Fisioterapia pela Faculdade Santa Maria, Cajazeiras PB - Email: vagner_2013bezzerra@hotmail.com.

${ }_{2}$ Fisioterapeuta. Graduado pela Faculdade Santa Maria, Cajazeiras-PB, E-mail: hiagoifce@gmail.com.

3 Fisioterapeuta. Mestre. Docente da Faculdade Santa Maria, Cajazeiras-PB, E-mail: julianecarlam@gmail.com.

Fisioterapeuta. Mestre. Docente da Faculdade Santa Maria, Cajazeiras-PB, E-mail: lullabraga@hotmail.com.

${ }^{5}$ Fisioterapeuta. Doutora. Docente da Universidade Federal de Campina Grande, Cajazeiras-PB, Email: ely.vilar@hotmail.com.

6 Fisioterapeuta. Mestre. Docente da Faculdade Santa Maria, Cajazeiras-PB, E-mail: michelj_dias@hotmail.com.
} 
todos os segmentos corporais, apontando a maior prevalência para a região da coluna vertebral e o nível da qualidade de sono foi considerado ruim.

Palavras chave: Postura. Saúde do trabalhador. Sono.

\section{INTRODUÇÃO}

O bombeiro militar $(B M)$ é um importante servidor público com extrema importância na nossa sociedade. E sua atuação profissional pode colocar em risco sua própria vida para poder salvar a vida de outra pessoa, bem como os patrimônios públicos ou privados da sociedade. O BM atua na defesa civil, prevenção e combate a incêndio, perícias técnicas, busca e salvamento, ou seja, de forma geral, estabelecer normas relacionadas à segurança das pessoas e de seus bens a qualquer tipo de catástrofe (VICENTE, FERREIRA, REZENDE, 2013).

A rotina de trabalho pode contribuir para ocorrência de alterações na saúde do mesmo. Esse fato pode ser causado em decorrência de várias situações onde estão inserido podendo estar expostos a agentes patológicos ou a causas que tenham vínculos relacionados a organização do trabalho, como distúrbios da função decorrente de procedimento operacional, desencadeando um trabalho para favorecer o alcance de melhor resultado (WORM et al., 2016).

Souza e colaboradores (2012) relatam o aumento do estado de alerta que está ligado de forma direta ao desgaste físico e psíquico, e percutindo assim nas formas de fadiga, e principalmente transtorno do sono devido a altas ocorrências de atuação, fazendo com que BM não tenham um bom sono, causando com isso diminuição na atuação do mesmo no que se refere a seu serviço de trabalhos prestados a comunidade.

Atividades que requerem uma postura prolongada estressam regiões corpóreas devido à contração dos grupos de músculos que estão atuando, a ponto de serem correspondido com pontos dolorosos. A união de aspectos motoresfuncionais com o tipo da ocupação dos BM, aspectos administrativos ao qual são forçados a executar nas ocorrências e fatores psicossociais, que são aliados no 
desenvolvimento de distúrbios funcionais, ou seja, quadros álgicos e lesões musculoesqueléticas (QUEIROGA, FERREIRA, 2005).

O sono, fenômeno essencial para a sobrevivência, tem como função primordial a restauração corporal e mental. Podendo ser afetado por diferentes fatores que interferem no ciclo sono-vigília, dentre eles: a privação do sono, presença de distúrbios mentais, efeitos de drogas no Sistema Nervoso Central (SNC), hábitos irregulares, idade, patologias físicas e cognitivas, mudança de fuso horário e ritmo circadiano (COELHO et al., 2010).

Alterações no sono pode ser um fator perturbador para o bombeiro militar, visto que, independente do seu período de descanso noturno se surgir alguma ocorrência esse descanso será interrompido, o qual acaba influenciando em incômodos perante a sua atuação. O sono regular tem um papel importante para a função normal do sistema endócrino e imunitário. O núcleo do sistema nervoso central (SNC) é sincronizado pela relação entre o ambiente físico e o corpo, produzindo e liberando hormônios de acordo com as situações vivenciadas (ESPACASSASSI, 2015).

Portanto, o presente trabalho se justifica na compreensão de avaliação postural e do sono como elementos fundamentais no bom desempenho das funções do bombeiro militar, uma vez que essa temática pode fornecer ações que podem ser desenvolvidas para o trabalhador dentro do ambiente do seu cotidiano.

O objetivo desse estudo foi avaliar a postura e os aspectos do sono dos bombeiros militares da cidade de Cajazeiras-PB.

\section{MÉTODO}

O presente estudo caracteriza-se por uma pesquisa de natureza analítica, transversal com abordagem quantitativa. $O$ estudo foi constituído por 20 bombeiros e a amostra se deu por conveniência e aleatória. A pesquisa foi realizada $5^{\circ}$ batalhão do corpo de bombeiro situado Av. Conte Vital Rolim, 1044 - Jardim 
Adalgiza II, Cajazeiras - PB, 58900-000, desenvolvida nos meses de agosto e setembro de 2018.

Foram incluídos os BM que exercem atividades e que atuem no $5^{\circ}$ Batalhão do corpo de bombeiros, acima de 18 anos de idade e de ambos os sexos. E excluídos do estudo, os que estavam de férias, afastados das atividades, e os que não estiverem presentes no dia da coleta.

Para avaliação do sono foi utilizado o questionário do índice de qualidade do sono de Pittsburgh (PSQI) no (anexo A). O PSQI avalia a qualidade e perturbações do sono durante o período de um mês e foi desenvolvido por Buysse et al. (1989). instrumento é constituído por 19 questões em auto-relatadas e cinco questões direcionadas ao cônjuge ou acompanhante de quarto. As últimas cinco questões são utilizadas apenas para a prática clínica.

E para a análise postural foi pedido autorização dos dados obtidos pelo setor administrativo através do programa AVAESPORTE versão 2.0.14. É um software de avaliação física, prescrição de treino e gestão administrativa, desenvolvido na Universidade Federal de Viçosa (UFV), que vem se tornando a principal ferramenta dos profissionais de educação física, fisioterapia, nutrição e medicina esportiva, pois facilita e agiliza o seu dia a dia de trabalho e traz maior qualidade para o resultado apresentado.

A pesquisa foi realizada de manhã e à tarde, três vezes por semana, nos horários livres e de descanso dos BM. O preenchimento se deu de forma individualizada, em uma sala disponibilizada especificamente para a coleta dos dados, com duração de 25 minutos. Após a finalização dos questionários, foram coletados os dados estatísticos do AVAESPORTE.

A análise seguiu o conforme o escore de PSQI aonde foi atribuído uma nota para cada componente e somado cada componente para se chegar ao resultado esperado com a soma de cada componente para se chegar ao escore final, o qual define se o bombeiro militar tem ou não distúrbio do sono. Os testes de KolmogorovSmirnov, Shapiro Wilk e Levene foram utilizados para verificar a normalidade e homogeneidade dos dados. E adotado um intervalo de confiança de 95\%. O estudo ocorreu conforme aprovação do estudo pelo Comitê de Ética e Pesquisa (CEP) da Faculdade Santa Maria (FSM), com o número do parecer: 2.608.355 (ANEXO B), e 
seguiu as normas da resolução 466/12 do Conselho Nacional de Saúde - Pesquisa envolvendo seres humanos (BRASIL, 2012).

\section{RESULTADOS E DISCUSSÃO}

A amostra do estudo foi composta por 20 (vinte) bombeiros militares, com prevalência para o sexo masculino, totalizando $90 \%$. Com relação à idade, a predominância foi para os participantes que se enquadravam na categoria de 26 a 35 anos.

Quanto o grau de escolaridade, 35\% dos respondentes afirmaram possuir ensino superior completo, e no que diz respeito ao estado civil, grande parte dos participantes relataram ser casado.

Em relação aos turnos de trabalho, $80 \%$ relataram trabalhar manhã, tarde e noite. Com relação à jornada de trabalho, 80\% evidenciaram trabalhar acima de 12 horas de trabalho. Com relação aos dias da semana de trabalho, 70\% relataram trabalhar nove dias ininterruptos/ 27 dias de folga. E sobre as horas de descanso $45 \%$ dos entrevistados afirmaram que essas horas eram indefinidas.

Além da carga horária de trabalho com média de 24 horas cada plantão, os BM têm um trabalho árduo dentro das horas já mencionadas, o que ocasiona efeitos prejudiciais a sua condição de saúde, uma vez que isso pode interferir em sua saúde. Este trabalho excessivo e pouco remunerado pode favorecer o aparecimento de sintomas osteomusculares (BRANCO 2014).

A sobrecarga de trabalho está ligada a problemas achados no meio profissional, como também fora do batalhão, dentre as quais se pode citar as várias jornadas e aumento das horas de trabalho, aumento do número de ocorrências na comunidade, locais de ocorrências em condições precárias, sem tirar a falta de profissionais nas outras áreas, e pôr fim a falta de reconhecimento do trabalho feito pela sociedade (GOMES, 2002).

Condições de trabalho são as circunstâncias em que o trabalho é realizado e compreendem tanto aspectos do ambiente quanto da sua organização. Quando as 
condições são precárias e o volume de atividades se torna excessivo, exigindo altas demandas físicas e emocionais, acabam comprometendo a eficiência do BM. Além disso, o cenário de condições precárias para o trabalho acaba por exigir dos BM um sobre-esforço ou uma hipersolicitação de suas funções psicofisiológicas. A quantidade de tarefas executadas representa demandas ou exigências psicobiológicas do processo de trabalho que levam ao desgaste do trabalhador (GUERREIRO, 2016).

Sobre as questões relacionadas ao sono, associado à qualidade subjetiva prevalece com 55\% a variável boa qualidade do sono; já em relação à latência do sono predomina com 70\%; em relação à duração do sono observa-se que $50 \%$ dos bombeiros tem uma média de sono entre 6-7 horas, e em sobre os distúrbios do sono $55 \%$ dos bombeiros avaliados apresentam essa alteração.

Observa-se na tabela 1 que o componente que apresentou maior índice no presente estudo foi o de Latência do Sono, o menor, foi o uso de medicação para dormir, os respectivos desvios padrões de ambos mostra uma pequena variação nos resultados, indicando assim que os valores se desviam pouco em relação ao índice médio.

Tabela 1 - Caracterização das dimensões do índice de qualidade de sono.

\begin{tabular}{lcccc}
\hline $\begin{array}{c}\text { Dimensões de qualidade de } \\
\text { sono }\end{array}$ & Mínimo & Máximo & Média & Desvio Padrão \\
\hline Qualidade Subjetiva do Sono & 0 & 3 & 1,3 & 0,73 \\
Latência do sono & 0 & 3 & 1,5 & 0,94 \\
Duração do Sono & 0 & 3 & 1,2 & 0,89 \\
Eficiência do Sono & 0 & 3 & 1,3 & 1,22 \\
Perturbações do Sono & 0 & 3 & 1,4 & 0,68 \\
Uso de Medicação para Dormir & 0 & 2 & 0,1 & 0,48 \\
Disfunção durante o dia & 0 & 3 & 1,3 & 0,93 \\
Índice de Qualidade de Sono & $\mathbf{3}$ & $\mathbf{1 6}$ & $\mathbf{8 , 2}$ & $\mathbf{3 , 5}$ \\
\hline
\end{tabular}

Fonte: Dados da pesquisa, 2018.

A presença de distúrbio de sono evidenciada é um fator indicado em vários estudos sobre saúde do trabalhador. E para Calixto (2015) a presença de distúrbio do sono tem predileção principalmente nos praças que são mais fadigados pelo 
trabalho árduo de execução que apresentam maior prevalência de interferência na capacidade funcional.

Dentre os incômodos relatados pelos BM, destaca-se a dificuldade para descansar por conta das ocorrências. O trabalho em posições inadequadas pode, também, estar relacionado ao mobiliário disponível no local de trabalho, que muitas vezes são inadequados (SILVA; SILVA, 2013). Mais da metade dos afastamentos do trabalho dos bombeiros militares possuem como causa quadros de desgaste físico, dessa forma, faz-se necessário um olhar especial para a compreensão do adoecimento nessa categoria profissional. Esse processo, em conjunto com a formação deficitária, considerando-se a realidade nos batalhões (PEREIRA, 2014). Com relação ao grau de alterações do sono encontrado nos bombeiros militares, prevaleceu a categoria "Presença de distúrbios de sono" com uma porcentagem de $40 \%$, seguida da categoria "ruim" com o mesmo percentual $40 \%$, e por fim a categoria boa com $20 \%$.

Observa-se na vista anterior à predominância da inclinação a direita da cabeça $25 \%$, elevação do ombro à esquerda $65 \%$, cristas ilíacas assimétricas $80 \%$ e joelhos genovalgo com 35\%. (Tabela 2).

Tabela 2 - Alterações posturais encontradas na vista anterior, Cajazeiras-PB.

\begin{tabular}{cccc}
\hline SEGUIMENTOS & ALTERAÇÕES & $\mathbf{n}$ & $\%$ \\
\hline Cabeça & Inclinado D & $\mathbf{5}$ & $\mathbf{2 5}$ \\
Ombro & Inclinado E & 2 & 10 \\
& Elevado D & 7 & 3 \\
Crista ilíaca & Elevado E & $\mathbf{1 3}$ & 65 \\
Joelho & Assimétrico & $\mathbf{1 6}$ & 80 \\
& Genovalgo & $\mathbf{7}$ & $\mathbf{3 5}$ \\
& Genovaro & 2 & 10 \\
\hline
\end{tabular}

Fonte: Dados da pesquisa, 2018.

Observa-se na vista lateral a predominância da cabeça projetada para frente $50 \%$, protrusão do ombro 50\%, anteversão pélvica $65 \%$ e joelho genorecurvato com 35\%. (Tabela 3). 
Tabela 3 - Alterações posturais encontradas na vista lateral, Cajazeiras-PB.

\begin{tabular}{ccc|c}
\hline SEGUIMENTOS & ALTERAÇÕES & $\mathbf{N}$ & $\%$ \\
\hline Cabeça & Projetada p/ frente & $\mathbf{1 0}$ & $\mathbf{5 0}$ \\
Ombro & Projetada p/ trás & 3 & 15 \\
& Protuso & $\mathbf{1 0}$ & $\mathbf{5 0}$ \\
& Retraído & 7 & 35 \\
& & & \\
Crista ilíaca & Anteversão & $\mathbf{1 3}$ & $\mathbf{6 5}$ \\
Joelho & Retroversão & 2 & 10 \\
& Genorecurvato & $\mathbf{7}$ & $\mathbf{3 5}$ \\
\hline
\end{tabular}

Fonte: Dados da pesquisa, 2018.

$\mathrm{Na}$ vista posterior, as principais alterações posturais encontradas no segmento do ombro foram, 12 indivíduos com escápulas aladas, representando $60 \%$ dos avaliados, e 5 indivíduos com escápula retraída, representando 25\%. No segmento das pregas glúteas, 8 indivíduos se mostraram com assimetria à $D$ com $40 \%$ e 5 indivíduos com assimetria à $E$ com $25 \%$. A predominância foi escápulas aladas $60 \%$ e pregas glúteas assimétricas à direita com $40 \%$.

Para Souza et al. (2012) a dinâmica trabalho-saúde tem sido discutida nos grupos que completa o campo da saúde coletiva, especificadamente na área da saúde ocupacional do trabalho. $\mathrm{O}$ trabalho prescrito está diretamente sujeito às normas previstas pela organização do trabalho e os estudos, com base na ergonomia da atividade, partem do princípio de que as atividades sem descanso, as condições de trabalho e as tarefas que propiciam as posturas incorretas podem ser deletérias para a saúde dos trabalhadores.

\section{CONCLUSÃO}

O estudo evidenciou alterações posturais e as mais prevalentes foram: escápulas aladas, crista ilíaca assimétrica e hipercifose torácica. E o nível da 
qualidade de sono foi considerado ruim, com um percentual considerado de distúrbio do sono.

É possível observar que os bombeiros militares estão predispostos a vários fatores estressores, assim, favorecendo o prejuízo na sua saúde, consequentemente na sua qualidade de vida.

\section{REFERÊNCIAS BIBLIOGRÁFICAS}

VICENTE, N.G., FERREIRA, L.A.; REZENDE, M.P. Percepção do estresse ocupacional por bombeiros militares de uma cidade do interior de minas gerais. J. res.: fundam. care. Online, Minas Gerais, v.5, n.3, p.75, 2013.

WORM, F.A.; PINTO, M.A.; SCHIAVENATO, D.; ASCARI, R.A.; TRINDADE, L.; SILVA, O.M. Risco de adoecimento dos profissionais de enfermagem no trabalho em atendimento móvel de urgência. Rev. Cuid. Bahia, v.7, n.2, p.329, 2016.

QUEIROGA, M. R.; FERREIRA, S. A. Ocorrência de dor na coluna vertebral em motoristas de ônibus e bombeiros militares. UNOPAR Cient., Ciênc. Biol. Saúde. Londrina, n. 1, v.7, p. 21 26, out. 2005.

COELHO, A. T et al. Qualidade de Sono, Depressão e Ansiedade em Universitários dos Últimos Semestres de Cursos da Área da Saúde. R. neurobiologia, São Paulo. v.1, n. 73, p.35, jan./mar., 2010.

ESPACASSASSI. Avaliação hemodinâmica, estresse, perfil metabólico e balanço autonômico em profissionais bombeiro militar. Tese programa de pós-graduação em medicina. São Paulo, 2015.

BRASIL. Ministério da Saúde. Resolução 466/12 do Conselho Nacional de Saúde, sobre as diretrizes e normas regulamentadoras de pesquisa envolvendo seres humanos. Diário oficial da união, 13 de junho de 2012.

BRANCO, J. C. et al. Prevalência de Sintomas Osteomusculares em bombeiros militares. Revista Fisioterapia e Movimento, v.24, n.2, p.307-314, abr/jun, 2014.

GOMES, L. Trabalho multifacetado de bombeiros/as: a saúde entre limites. Fundação Oswaldo Cruz, Rio de Janeiro, 2002.

GUERREIRO, N. P. et al. Perfil Sociodemográfico, Condições e Cargas de Trabalho de Profissionais da Rede Estadual de bombeiros da região Sul do Brasil. Revista Educação, Trabalho e Saúde, v.14, n.1, p. 197-217, Rio Grande do Sul, 2016.

CALIXTO, M. F et al. Prevalência de Sintomas Osteomusculares e Suas Relações Com o Desempenho Ocupacional Entre Profissionais bombeiros. Cadernos de Terapia Ocupacional, v.23, n.3, p. 533-542, 2015.

SILVA, L. G.; SILVA, M. C. Condições de Trabalho e Saúde de bombeiros militares, RS, Brasil. Revista Ciência \& Saúde Coletiva, v.18, n.11, p.3137-3146, Rio Grande do Sul, 2013. 